\title{
Effects of the onabotulinumtoxinA follow-up delay in migraine course during the COVID-19 lockdown
}

\author{
Alicia Gonzalez-Martinez ${ }^{1,2}$ - Álvaro Planchuelo-Gómez ${ }^{3}$ Ángel L Guerrero ${ }^{4,5,6}$ • David García-Azorín ${ }^{4,5}$ (D) \\ Sonia Santos-Lasaosa ${ }^{7} \cdot$ María Pilar Navarro-Pérez ${ }^{7} \cdot$ Paula Odriozola-González $^{8}$ • María Jesús Irurtia ${ }^{8}$. \\ Sonia Quintas ${ }^{1,2}$. Rodrigo de Luis-García ${ }^{3}$. Ana Beatriz Gago-Veiga ${ }^{1,2}$
}

Received: 6 September 2020 / Accepted: 13 March 2021 / Published online: 26 March 2021

(C) Fondazione Società Italiana di Neurologia 2021

\begin{abstract}
Background Face-to-face procedures have been postponed during COVID-19 pandemic. We aim to evaluate the impact of onabotulinumtoxinA follow-up delay in migraine during COVID-19 pandemic.

Methods Subjective worsening, intensity of migraine attacks, and frequency of headache and migraine were retrospectively compared between patients with unmodified and interrupted onabotulinumtoxinA follow-up in Headache Units.

Results We included 67 patients with chronic migraine or high-frequency episodic migraine under onabotulinumtoxinA treatment, $65(97.0 \%)$ female, $44.5 \pm 12.1$ years old. Treatment administration was voluntarily delayed in $14(20.9 \%)$ patients and nine $(13.4 \%)$ were unable to continue follow-up. Patients with uninterrupted follow-up during lockdown presented 7.6 and 8.1 less monthly days with headache (adjusted $p=0.017$ ) and migraine attacks (adjusted $p=0.009$ ) compared to patients whose follow-up was interrupted, respectively.

Conclusion Involuntary delay of onabotulinumtoxinA follow-up in patients with migraine due to COVID-19 pandemic was associated with a higher frequency of headache and migraine attacks. Safe administration of onabotulinumtoxinA during lockdown should be promoted.
\end{abstract}

Keywords OnabotulinumtoxinA $\cdot$ Headache $\cdot$ Lockdown $\cdot$ Migraine $\cdot$ COVID-19 $\cdot$ SARS-CoV-2 $\cdot$ Follow-up

\section{Introduction}

COVID-19 pandemic has led to a change in headache clinical practice. There has been a marked reduction in the number of in-person visits as well as in-person treatments [1] in order to reduce widespread infections $[2,3]$. Consequently,

Alicia Gonzalez-Martinez and Álvaro Planchuelo-Gómez contributed equally to the manuscript.

David García-Azorín

davilink@hotmail.com

$1 \quad$ Headache Unit, Department of Neurology, Hospital Universitario de La Princesa and Instituto de Investigación Sanitaria de La Princesa, Madrid, Spain

2 Department of Neurology, Universidad Autónoma de Madrid, Madrid, Spain

3 Imaging Processing Laboratory, Universidad de Valladolid, Valladolid, Spain telemedicine visits have increased during the pandemic [4]. Moreover, it has been reported that patients with migraine under treatment with monoclonal antibodies have decided to continue with the treatment despite mobility restrictions [5], reflecting the burden of disease. However, the number of faceto-face appointments for in-person treatments such as
4 Headache Unit, Department of Neurology, Hospital Clínico Universitario de Valladolid, Valladolid, Spain

5 Institute for Biomedical Research of Salamanca (IBSAL), Salamanca, Spain

6 Department of Medicine, Universidad de Valladolid, Valladolid, Spain

7 Headache Unit, Department of Neurology, Hospital Clínico Universitario Lozano Blesa and Aragon Institute for Health Research (IIS Aragón), Zaragoza, Spain

8 Department of Psychology, Universidad de Valladolid, Valladolid, Spain 
onabotulinumtoxinA have been drastically reduced. To date, little is known about the impact of onabotulinumtoxinA delay in the migraine course during the pandemic and lockdown. The objective of this study was to determine whether missing in-person appointments for the onabotulinumtoxinA planned follow-up have resulted in a resurgence of migraine headaches during the COVID-19 lockdown.

\section{Methods}

A retrospective cross-sectional study comparing migraine course in patients treated with onabotulinumtoxinA before and during the COVID-19 lockdown was carried out. Four characteristics were evaluated during the lockdown: subjective worsening of the migraine, intensity of the migraine attacks, and number of monthly days with headache and migraine. Number of monthly days with headache contained any type of headache, including migraine, and another variable was considered for the days with migraine.

The study population were patients with migraine under onabotulinumtoxinA treatment that were followed-up at the Headache Units of three Spanish academic hospitals. The inclusion criteria were diagnosis of migraine according to the third edition of the International Classification of Headache Disorders, $3^{\text {rd }}$ edition (ICHD-3) [6] under treatment with onabotulinumtoxinA, aged over 17 years, and willing to participate in the study. Patients were excluded if they suffered from serious neurological or systemic disease, confirmed or suspected positive COVID-19 diagnosis, and any headache disorder different to migraine, infrequent tension-type headache, or medication overuse headache. Patients were invited to participate in the study after telephone contact or in-person appointment. After verbal consent was obtained, patients described the frequency and intensity of headache episodes by using a calendar and a headache diary and were asked to complete an online survey about demographic and clinical data, including personal migraine triggers and aggravating factors. The intensity of migraine attacks was measured using a numerical rating scale (1: lowest intensity; 10: highest intensity) [7]. Moreover, the participants were asked about symptoms of post-traumatic stress at the moment that the survey was answered. The psychological impact specifically associated with the COVID-19 lockdown was evaluated using the Impact of Event Scale (IES) [8]. This study was approved by the Ethics Review Board of Valladolid East health area.

\section{Statistical analysis}

Group proportions were obtained for the categorical (nominal and ordinal) variables. For the quantitative variables (interval and ratio), mean and standard deviation were calculated. For the analysis of subjective worsening of migraine, a logistic regression model was employed, while the other three characteristics (intensity of migraine attacks and number of monthly days with migraine and headache) were evaluated with a Generalized Linear Model (GLM) following a Gaussian distribution, using as response variable the difference between the numeric value during the lockdown and the value before the lockdown. The value selected for the lockdown period was the value in the last month being confined, and the value for the previous period was the median value of the 3 months before the lockdown. The multivariate model, including the values of the other evaluated variables commented in the last paragraph before this subsection, was obtained using an automatic stepwise procedure and the lowest Akaike's Information Criteria value as stop criterion for the selection of the final model. The statistical significance threshold was a $p$ value of 0.05 , after the comparison for multiple correction using a false discovery rate method. We performed no previous estimation of the sample size. Patients with missed answers related to their onabotulinumtoxinA treatment were not included in the study. $\mathrm{R}$ statistical software version 3.5.2. was employed.

\section{Results}

A total of 68 patients under onabotulinumtoxinA treatment were initially recruited for this study; however, one of these patients gave no answer about the follow-up during the lockdown period and was discarded. Among the remaining 67 patients, 65 (97.0\%) were women, aged $44.5 \pm 12.1$.

Fourteen $(20.9 \%)$ patients suffered a voluntary delay in treatment administration and nine (13.4\%) were unable to make a follow-up during the COVID-19 pandemic. Among patients who experienced a delay or were unable to receive onabotulinumtoxinA administration 12/23 (52.2\%) experienced subjective worsening, 7/23 (30.4\%) increase in the intensity of migraine attacks, and 9/23 (39.1\%) increase in both monthly number of days with migraine and headache attacks. The main demographic, clinical, and changes in the onabotulinumtoxinA follow-up and the analyzed variables related to migraine are summarized in Table 1. Figure 1 shows the changes of intensity of migraine attacks, and frequency of headache and migraine during the lockdown, in the three groups of assessed patients.

No significant differences in the subjective worsening of migraine and the intensity of migraine attacks were found between the three groups during the COVID-19 lockdown.

With respect to number of monthly days with headache, compared to the patients who had no chance to continue their follow-up during the COVID-19 lockdown, a significant decrease between 7 and 9 days per month was observed in the patients who voluntarily delayed their treatment $(\beta=-8.7$, corrected $p=0.017$ ) and those who continued with the 
Table 1 Demographic characteristics of patients under onabotulinumtoxinA treatment

\begin{tabular}{lll}
\hline Characteristics & Number (\%) & Mean \pm SD \\
\hline Age (years) & N/A & $44.5 \pm 12.1$ \\
Sex & & N/A \\
Male & $2(3.0)$ & \\
Female & $65(97.0)$ & $23.3 \pm 12.7$ \\
Duration of migraine (years) & N/A & $13.5 \pm 8.2$ \\
Frequency of headache before the lockdown (days/month) & N/A & $10.5 \pm 7.3$ \\
Frequency of migraine before the lockdown (days/month) & N/A & N/A \\
Follow-up of onabotulinumtoxin-A & & \\
Unable to go to in-person administration & $9(13.4)$ & \\
Voluntary delay & $14(20.9)$ & \\
Normal in-person follow-up & $44(65.7)$ & \\
\hline
\end{tabular}

N/A not applicable planned follow-up $(\beta=-7.6$, corrected $p=0.017)$. The previous results are adjusted by the effect of duration of migraine, the use of medication for anxiety and depression, menstruation, and IES scores. A significant higher frequency of headache was found in patients with higher duration of migraine ( $\beta$ $=0.2$, corrected $p=0.040)$. In patients with prescribed medication for depression, higher frequency of headache was found, although there was no statistical significance after correction for multiple comparisons $(\beta=5.0$, uncorrected $p=$ $0.031)$.

Regarding the migraine frequency, compared to the patients with involuntary delayed follow-up, a reduction of 8-9 days per month in patients with the planned onabotulinumtoxinA follow-up $(\beta=-8.1$, corrected $p=$ 0.009 ) and with voluntary delay of the treatment administration $(\beta=-8.4$, corrected $p=0.011)$ was also found. The previous results are adjusted by the effect of sex, diagnosis of anxiety and depression during lockdown, previous diagnosis of anxiety, the use of medication for depression, diverse reported migraine triggers (menstruation, sleep disturbances, number of hours working, and work pressure), and changes in any of the personal aggravating factors of migraine. A lower number of monthly migraine attacks was related to menstruation as migraine trigger $(\beta=-7.5$, corrected $p=0.001)$ and changes in aggravating factors $(\beta=-5.9$, corrected $p=$ 0.011). The significant results are shown in Fig. 2.

\section{Discussion}

In the present study we assessed the impact of changes in the onabotulinumtoxin A administration schedule, as one of the few treatments that could not be replaced by telemedicine during the pandemic. Patients experiencing involuntary delay in onabotulinumtoxin A administration during the pandemic have suffered a remarkably higher number of monthly days with headache and migraine attacks. This report supports that postponing not essential procedures [4], such as onabotulinumtoxinA injections [9], has a negative impact in high-frequency episodic and chronic migraine patients.

According to our results, the patients who could continue with the expected onabotulinumtoxinA follow-up suffered from headache or migraine attacks between 7.5 and 8.5 days per month less in comparison with the patients who were unable to go to the in-person appointment. These results are supported by a recent study evaluating the effects of botulinumtoxin delay in patients with other pain conditions such as dystonia, spasticity, or hemifacial spasm, demonstrating the burden of neurological diseases during the lockdown [10]. Moreover, the botulinumtoxin delay in neurological patients has also been related to self-perceived worsening of their condition during the COVID-19 lockdown compared to controls [11]. In the same direction, our results demonstrate that onabotulinumtoxinA delay has also caused downside effects on patients with other chronic pain conditions such as chronic migraine. All together, these results suggest that neurological disease in need of botulinumtoxin in-person treatment administration could dramatically worsen during the lockdown. This information should be taken into account when making decisions about limiting access to treatments during the lockdown. Improving access to botulinumtoxin treatment for patients under chronic pain or disabling neurological conditions could avoid preventable worsening of patients in the case of a lockdown.

Interestingly, a very similar result with respect to the patients with the normal follow-up was obtained with those patients who voluntarily delayed their appointment. It is worth noting that almost two thirds the patients who delayed their appointment $(9 / 14 ; 64.3 \%)$ reported that they were feeling good when the appointment was set and, considering the lockdown situation and their personal well-being at that moment, they preferred not to go to the Headache Unit. 

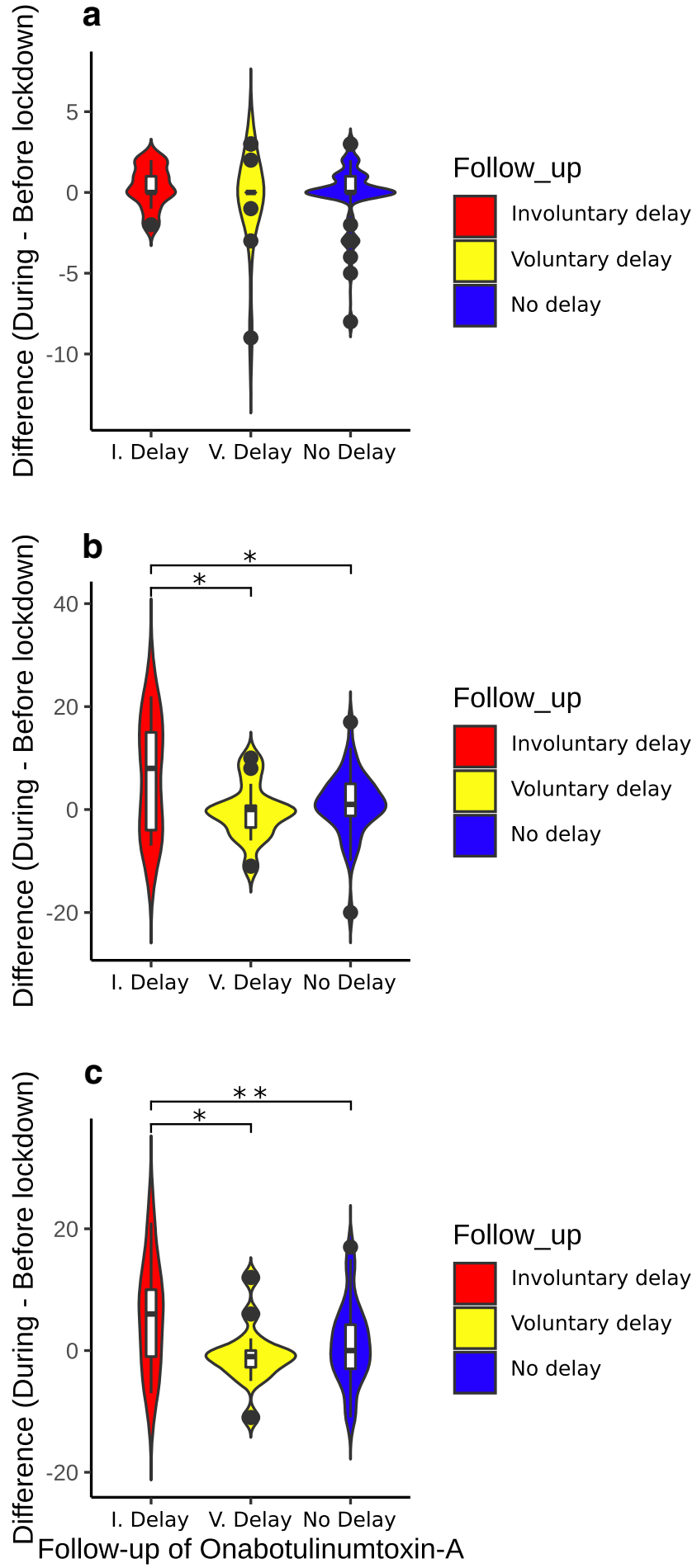

Fig. 1 Follow up of onabotulinumtoxinA. a Changes of intensity of migraine attacks, and $\mathbf{b}$ frequency of headache and $\mathbf{c}$ migraine during the lockdown

In addition to the follow-up of the onabotulinumtoxinA, the need of anti-depressant treatment also influenced the frequency of headache during lockdown. This result supports that psychiatric comorbidities such as depression influence onabotulinumtoxinA treatment effect [12, 13].
Regarding additional factors influencing the migraine frequency in patients with onabotulinumtoxinA, patients who reported menstruation as a migraine trigger were found to present a lower number of monthly migraine days. Hence, our results may suggest that women might have managed better the migraine related to menstruation while staying at home [14].

With respect to changes in aggravating factors, our results showed that these variations were associated with fewer number of migraine attacks per month. However, any significant difference was found neither for the subjective worsening of migraine nor the intensity of migraine attacks between the three assessed groups. Depending on the personal situation of the patients, particularly related to stress, working, and quality of sleep, frequency of headache could vary differently. In association with this personal situation, it has been reported that quality of life has been reduced in patients with chronic migraine during the COVID-19 lockdown in comparison with healthy family members (controls) [15]. Despite aggravating factors such as stress, exposure to work, and poor quality of sleep, the onabotulinumtoxinA treatment presented a positive effect in the patients with the planned follow-up compared to those with involuntary delay.

In addition to the aggravating factors, it must be considered that the onabotulinumtoxinA delay may have caused adverse effects such as anger or despair, related to anxiety, stress, and depression. It has been reported that patients experimenting a delay in botulinumtoxin administration during the lockdown found that their rights were not respected and found irritating that decision makers did not find their pain conditions severe enough to trigger treatment [10]. Patients with migraine are over 2.5 times more likely to suffer from depression compared to healthy controls, and depression has been stated as a predictor of migraine chronification and has been associated with medication overuse and being refractory to migraine treatments [16]. According to our findings, the patients with a prescribed treatment for depression presented approximately five additional monthly days during the lockdown compared to those with no treatment, although this results was not statistically significant after corrections for multiple comparisons. Depression is often comorbid with anxiety disorders in migraine [17]. The control of anxiety has been linked to improved quality of life and effectiveness of migraine treatment [17]. In relation to depression, stressful events have been reported to increase the incidence and susceptibility of major depressive disorder and other psychiatric comorbidities [16]. Thus, considering that anxiety, depression, and especially stress levels have increased throughout the lockdown related to the COVID-19 pandemic [18], the onabotulinumtoxinA follow-up would be important not only to reduce frequency of headache and migraine but also to prevent a worsening of the clinical course associated with the psychiatric comorbidity. In relation to the positive effects of an appropriate followup, in patients with migraine receiving monthly erenumab or 
Fig. 2 Comparison of characteristics (changes during lockdown). a Frequency of headache. b Frequency of migraine

\section{Comparison of characteristics (changes during lockdown)}

a

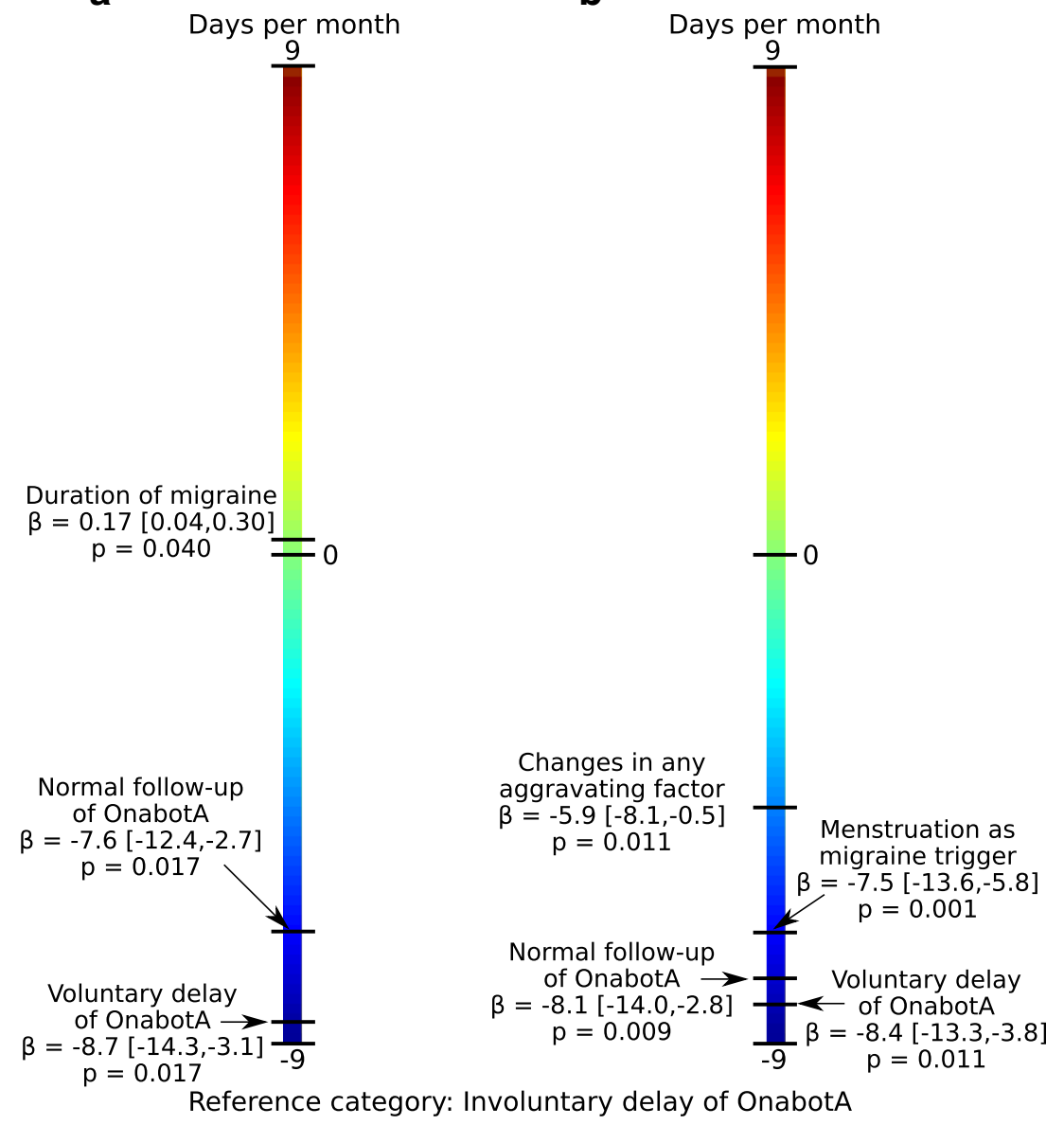

galcanezumab it has been reported a statistically significant decrease of monthly migraine days despite the lockdown [19].

The main limitation of this study was the retrospective analysis and a possible recall bias considering that the patients should remember the former state as better or worse, which could have influenced the subjective worsening analysis and other results. Another limitation of the study may be related to the lack of motivation of some patients to answer the survey, which may have biased the results. Regarding the delay of the onabotulinumtoxinA administration, we did not measure the number of days in the delay since the expected planned inperson visit with no treatment administration, which may have caused a negative impact in the frequency of headache and migraine. In relation to the symptomatic treatment, the frequency of its consumption was not collected, which may have influenced the analysis of patients who discontinued onabotulinumtoxinA. However, the exclusion of the patients with medication overuse headache reduced the possible impact of the frequency of symptomatic treatment use.

For an appropriate onabotulinumtoxinA follow-up, all safety measures should be guaranteed through the administration of proper medical equipment to protect the patients and neurologists.

\section{Conclusion}

Involuntary delay in onabotulinumtoxin A treatment in patients with migraine was associated with higher frequency of migraine attacks and headache. The negative impact of onabotulinumtoxinA delay in these patients might suggest that face-to-face treatment administration following safety recommendations could be a good option for management of chronic or high-frequency episodic migraine during lockdown.

\section{Clinical implications}

The follow-up of onabotulinumtoxinA during COVID-19 lockdown was interrupted in approximately a third of patients with migraine, with a negative effect in those patients with involuntary delay (approximately $40 \%$ of the patients with interrupted follow-up in our sample).

Patients with involuntarily interrupted follow-up presented 7-9 more monthly days with headache and migraine attacks compared to patients with voluntary delay and uninterrupted follow-up during COVID-19 lockdown. 
In-person onabotulinumtoxin A administration should be provided following safety recommendations in patients with chronic or high-frequency episodic migraine.

Supplementary Information The online version contains supplementary material available at https://doi.org/10.1007/s10072-021-05180-8.

\section{Declarations}

\section{Conflict of interest None.}

Ethical approval The study was approved by the Ethics Review Board of Valladolid East health area (PI 20-1832), based in the Hospital Clínico Universitario de Valladolid (Valladolid, Spain).

\section{References}

1. Ali A (2020) Delay in onabotulinumtoxinA treatment during the COVID-19 pandemic - perspectives from a virus hotspot. Headache. 60(6):1183-1186

2. López-Bravo A, García-Azorín D, Belvís R, González-Oria C, Latorre G, Santos-Lasaosa S et al (2020) Impact of the COVID19 pandemic on headache management in Spain: an analysis of the current situation and future perspectives. Neurol (Engl Ed) 35(6): 372-380

3. Santos-Lasaosa S, Porta-Etessam J (2020) OnabotulinumtoxinA infiltration and nerve blocks in patients with headache and neuralgia: safety recommendations to prevent SARS-CoV-2 infection. Neurol (Engl Ed) 35(5):291-294

4. Szperka CL, Ailani J, Barmherzig R, Klein BC, Minen MT, Halker Singh RB et al (2020) Migraine care in the era of COVID-19: clinical pearls and plea to insurers. Headache. 60(5):833-842

5. Silvestro M, Tessitore A, Tedeschi G, Russo A (2020) Migraine in the time of COVID-19. Headache. 60(5):988-989

6. Headache Classification Committee of the International Headache Society (2018) The International Classification of Headache Disorders, 3rd edition. Cephalalgia. 38(1):1-211
7. Loder E, Burch R (2012) Measuring pain intensity in headache trials: which scale to use? Cephalalgia. 32(3):179-182

8. Horowitz MJ, Wilner N, Alvarez W (1979) Impact of Event Scale: a measure of subjective stress. Psychosom Med 41(3):209-218

9. Porta-Etessam J, Gonzalez-Garcia N, Matias-Guiu JA, MonteroEscribano P, Matías-Guiu J (2020) Should we adapt the prescription criteria for specific treatments for migraine due to the COVID19 pandemic? Headache. 60(7):1448-1449

10. Dressler D, Adib SF (2020) Botulinum toxin therapy in the SARSCoV-2 pandemic: patient perceptions from a German cohort. J Neural Transm 127(9):1271-1274

11. Erro R, Scannapieco S, Russo M, Picillo M, Barone P (2021) Impact of COVID-19 on neurological patients attending a botulinum toxin service. Neurol Sci 42(2):433-435

12. Gonzalez-Martinez A, Rodríguez Vázquez E, de la Red Gallego H, García-Azorín D, Gallego de La Sacristana M, Guerrero Peral ÁL et al (2020) Association between personality traits and onabotulinumtoxinA response in patients with chronic migraine. Headache. 60(1):153-161

13. Boudreau GP, Grosberg BM, McAllister PJ, Lipton RB, Buse DC (2015) Prophylactic onabotulinumtoxinA in patients with chronic migraine and comorbid depression: an open-label, multicenter, pilot study of efficacy, safety and effect on headache-related disability, depression, and anxiety. Int J Gen Med 8:79-86

14. Marmura MJ (2018) Triggers, protectors, and predictors in episodic migraine. Curr Pain Headache Rep 22(12):81

15. Consonni M, Telesca A, Grazzi L, Cazzato D, Lauria G (2021) Life with chronic pain during COVID-19 lockdown: the case of patients with small fibre neuropathy and chronic migraine. Neurol Sci 42(2): 389-397

16. Minen MT, Begasse De Dhaem O, Kroon Van Diest A, Powers S, Schwedt TJ, Lipton R et al (2016) Migraine and its psychiatric comorbidities. J Neurol Neurosurg Psychiatry 87(7):741-749

17. Baskin SM, Lipchik GL, Smitherman TA (2006) Mood and anxiety disorders in chronic headache. Headache. 46(Suppl 3):S76-S87

18. Planchuelo-Gómez Á, Odriozola-González P, Irurtia MJ, de LuisGarcía R (2020) Longitudinal evaluation of the psychological impact of the COVID-19 crisis in Spain. J Affect Disord 277:842-849

19. Altamura C, Cevoli S, Aurilia C, Egeo G, Fofi L, Torelli P et al (2020) Locking down the CGRP pathway during the COVID-19 pandemic lockdown: the PandeMig study. Neurol Sci 41(12): 3385-3389

Publisher's note Springer Nature remains neutral with regard to jurisdictional claims in published maps and institutional affiliations. 